\title{
Recurrent Adrenal Gland Pheochromocytoma
}

National Cancer Institute

\section{Source}

National Cancer Institute. Recurrent Adrenal Gland Pheochromocytoma. NCI Thesaurus.

Code C8047.

The reemergence of a pheochromocytoma following surgical removal. 